\title{
Multiple focus and Hopf bifurcations in a predator prey system with nonmonotonic functional response
}

\author{
Dongmei Xiao * \\ Department of Mathematics \\ Shanghai Jiao Tong University, Shanghai 200030, China \\ Email: xiaodm@sjtu.edu.cn \\ Huaiping $\mathrm{Zhu}^{\dagger}$ \\ Laboratory of Industrial and Applied Mathematics \\ Department of Mathematics and Statistics \\ York University, Toronto, Ontario, Canada, M3J 1P3 \\ Email: huaiping@mathstat.yorku.ca
}

\begin{abstract}
In this paper, we develop a criterion to calculate the multiplicity of a multiple focus for general predator prey systems. As applications of this criterion, we calculate the most multiplicity of a multiple focus in a predator prey system with nonmonotonic functional response $p(x)=\frac{x}{a x^{2}+b x+1}$ studied by Zhu, Campbell and Wolkowicz [26] and prove that the degenerate Hopf bifurcation is of codimension two. Furthermore, we show that there exist parameter values for which this system has a unique positive hyperbolic stable equilibrium and exactly two limit cycles, the inner one is unstable and outer one is stable. Numerical simulations for the existence of the two limit cycles bifurcated from the multiple focus were also given in support of the criterion.
\end{abstract}

Keywords: Predator prey, Liénard system, multiple focus, Hopf bifurcation, codimension two, limit cycles

Primary 34C25, 92D25; Secondary 58F14

\section{Introduction}

The existence and number of limit cycles are important topics in the study of most applied mathematical models. It has been made possible of a better understanding of many real

*Research supported by the National Natural Science Foundations of China (No.10231020 ).

${ }^{\dagger}$ Corresponding author, Research supported by NSERC, CFI and MITACS of Canada. 
world oscillatory phenomena in nature $[1,11,17]$. For the predator-prey systems, it is wellknown that the existence of limit cycles is related to the existence, stability and bifurcation of a positive equilibrium. In a positively invariant region, if there exists a unique positive equilibrium which is unstable, then there must exist at least one limit cycle according to the theory of Poincare-Bendixson. On the other hand, if the unique positive equilibrium of a predator prey system is locally stable but not hyperbolic, there might be more than one limit cycles created via Hopf bifurcation(s). Numerical simulations of Hofbauer and So [8] indicated that it is indeed the case: there can exist at least two limit cycles for some two dimensional predator prey systems. It was proved by Zhu, Campbell and Wolkowicz [26] that the predator-prey system with nonmonotonic functional response can undergo a degenerate Hopf bifurcation which produces two limit cycles, and the system can also have two limit cycles through a saddle-node bifurcation of limit cycles. These two limit cycles can disappear through either supercritical Hopf or/and homoclinic bifurcations. Kuang [9] and Wrzosek [22] also observed that some predator-prey systems can even have more than two limit cycles. We shall point out that in all the models studied in $[8,9,22]$, the death rate of the predator is nonlinear but ours in $[19,26]$ is linear.

Hopf bifurcation theory is a powerful tool to study the existence, number and properties of the limit cycles in mathematical biology. However, the most number of limit cycles which can be created via a Hopf bifurcation is determined by the multiplicity of a multiple focus $[2,14]$ or correspondingly the codimension of the Hopf bifurcation. Therefore, the resolution of the multiplicity of a multiple focus plays a key role in determining the number of limit cycles for predator-prey systems.

In this paper, we first consider a general predator prey system, taken to have the form

$$
\left\{\begin{array}{l}
\dot{x}=a(x)-b(y) p(x), \\
\dot{y}=c(y) q(x)
\end{array}\right.
$$

which has a positive non-degenerate equilibrium in the first quadrant and it is a centertype equilibrium. For the predator prey system in the form of (1.1), it often involves the Hopf bifurcation(s) and it is essential to identify the multiplicity of a multiple focus in order to determine the codimension of the Hopf bifurcation. There are formulas available to calculate the Liapunov coefficients $[2,3,10,14]$ which can be used to decide the multiplicity, yet it usually becomes very challenging if the multiple focus has multiplicity great than or equal to two. In this paper, we shall first transform the general predator prey system (1.1) into a generalized Liénard-type system. Then a criterion will be established to calculate the multiplicity of a focus for the general predator prey system.

As applications of this criterion, following [26] we continue our study on the predatorprey systems with nonmonotonic functional response of the form

$$
\left\{\begin{aligned}
\dot{x} & =r x\left(1-\frac{x}{K}\right)-y p(x), \\
\dot{y} & =y(-d+c p(x))
\end{aligned}\right.
$$

where $x$ and $y$ are functions of time representing population densities of prey and predator, respectively; $r>0$ is the maximum growth rate and $K>0$ is the carrying capacity of 
the prey, $d>0$ is the death rate of the predator. The functional response is of Holling type IV, and

$$
p(x)=\frac{m x}{a x^{2}+b x+1} .
$$

Here we write $p(x)$ as in [26] with $b>-2 \sqrt{a}$ such that functional response $p(x)$ remains nonnegative for all $x \geq 0$. Note that if $b=0, p(x)$ is reduced to the function used in [19]. For detailed biological interpretation and motivation of the model, interested readers may consult the work $[19,26]$ and the references therein.

Global qualitative and bifurcation analysis by Rothe and Shafer [18] and Zhu, Campbell and Wolkowicz [26] show that system (1.2) with the functional response (1.3) has very interesting and rich dynamics. More precisely, by allowing $b$ to be negative but $b>-2 \sqrt{a}$, $p(x)$ is concave up for small values of $x>0$ as it is for the sigmoidal functional response. It was shown in [26] that there exists a Bogdanov-Takens bifurcation point of codimension 3 , which acts as an organizing center for the bifurcation diagram described in $(b, d, K)$ space with $d, K>0$ and $b>-2 \sqrt{a}$. Then the bifurcation sequences were given for parameters $(b, K)$ in each meaningful subregion of the $(b, K)$ plane and the death rate of the predator $d$ is varied. The bifurcation sequences involving Hopf bifurcations, homoclinic bifurcations as well as the saddle-node bifurcations of limit cycles are determined using information from the study of the Bogdanov-Takens bifurcation of codimension 3 and an exhaustive utilization of the geometry of isolines of the system. In particular, the Hopf bifurcation of codimension one was completely described in the parameter space $(b, d, K)$ with $d, K>0$ and $b>-2 \sqrt{a}$. Also, a curve segment, or the so called degenerate Hopf bifurcation curve was defined in [26]. But there is one thing missing: when the parameters take the value along the degenerate Hopf bifurcation curve, the order of the Hopf bifurcation or the multiplicity of the multiple focus were left unknown, and for the parameters $(b, K)$ in some subset, the bifurcation sequences were not complete (cf. Theorem 6.22 in $[26])$.

In this paper, we shall fill the gap of [26] and complete the study of the multiple focus and the degenerate Hopf bifurcation. We focus on the case when system (1.2) has a positive equilibrium. We shall prove that the equilibrium is a multiple focus of multiplicity at most two and can be exactly two. By using the Hopf bifurcation theorem, and combining the existing results regarding the Hopf bifurcations in [26], we shall give a complete description of bifurcation diagram for Hopf bifurcation of codimension two. Furthermore, we investigate the existence of exactly two limit cycles of system (1.2). To the best of our knowledge, there is no references in which the existence of exactly two limit cycles in any predator-prey system has been rigorously proved. The difficulty is that though there are theorems on the existence of exactly two limit cycles for certain Liénard-type system (Zhang [24] and Zhou [25]), they are not applicable to the predatorprey systems that may have two limit cycles.

This paper is organized as follows. In section 2 we develop a criterion of the multiplicity of a multiple focus for the general system (1.1). System (1.2) with the functional response (1.3) is a typical example of the general predator-prey system with nonmontonic functional response. As applications, we will study (1.2) by focusing on the calculation of the multiplicity of the multiple focus and the description of the degenerate Hopf bifurcation. 
We shall prove that the multiplicity of the unique multiple focus is at most two and there exist parameter values such that system (1.2) with the functional response (1.3) has a multiple focus whose multiplicity is exactly two. We shall also prove that system (1.2) with the functional response (1.3) has exactly two limit cycles for some parameter values. A Hopf bifurcation diagram involving the Hopf bifurcation of codimension two will also be given based on the results related to the Hopf bifurcation of codimension one from [26]. We end section 3 by giving the numerical simulations of the two limit cycles. The paper ends with a brief discussion.

\section{The multiplicity of a multiple focus in a general predator-prey system}

In [21] Wolkowicz proposed a general form of the predator-prey model for studying the impact of the group defence. And Kazarinoff and van den Driessche in [13] studied a predator-prey system by incorporating a fairly general functional response. Here, we consider a general predator prey system of the form

$$
\left\{\begin{array}{l}
\dot{x}=a(x)-b(y) p(x), \quad x(0) \geq 0 \\
\dot{y}=c(y) q(x), \quad y(0) \geq 0
\end{array}\right.
$$

where $x$ and $y$ are functions of $t$ representing the density of prey and predator populations at a given time $t \geq 0$, respectively. Basing on the biological meaning, we will restrict ourselves to the first quadrant and in particular, we make the following assumptions:

(A1) the growth function of the prey $a(x)$ is $C^{1}$ for $x \in[0,+\infty)$ and $a(0)=0$;

$(\mathrm{A} 2) b(y), c(y) \in C^{1}[0,+\infty), b(0)=c(0)=0$ and $b^{\prime}(y)>0, c^{\prime}(y)>0$ for all $y \geq 0$

(A3) $p(x) \in C^{1}[0,+\infty), p(0)=0$ and $p(x)>0$ for all $x \geq 0$;

(A4) $q(x) \in C^{1}[0,+\infty)$, and there exists $x_{0}>0$ such that

$$
q\left(x_{0}\right)=0, \quad a\left(x_{0}\right) / p\left(x_{0}\right)>0 \text { and } q^{\prime}\left(x_{0}\right)>0 .
$$

With the above assumptions, one can verify that both the $x$ and $y$-axes are invariant. Therefore, the first quadrant is positively invariant. Furthermore, a straightforward computation can verify that if the assumptions (A1)-(A4) hold, then system (2.1) has a positive equilibrium $\left(x_{0}, y_{0}\right)$, which is non-degenerate, where $y_{0}=b^{-1}\left(a\left(x_{0}\right) / p\left(x_{0}\right)\right)$ and $b^{-1}$ is the inverse function of $b(y)$. Thus, there exists a neighborhood $\Omega$ of $\left(x_{0}, y_{0}\right)$ in the first quadrant such that system (2.1) has no other equilibria except $\left(x_{0}, y_{0}\right)$ in $\Omega$. It is clear that in the generic case any predator prey system can have a positive equilibrium, which is a focus or a center or a node or a saddle point. Note that by (A2) we have $c\left(y_{0}\right)>0$, hence in order for the positive equilibrium $\left(x_{0}, y_{0}\right)$ to be a center-type focus, we have to further assume that 
Then, under the assumptions (A1)-(A5), system (2.1) has an isolated positive equilibrium $\left(x_{0}, y_{0}\right)$ in $\Omega$, which is a center-type equilibrium.

Recall that many of the classical predator-prey systems can be written in the form of (2.1) with assumptions (A1)-(A5) satisfied. For example, the predator-prey system with response function of Holling types falls into this category [6, 19, 13, 21, 26]. There have been extensive studies on the stability and bifurcations for the predator-prey systems (see $[8,9,18,19,21,22,23,26]$ and references therein), but it is not an easy task to study the number of limit cycles which can be born through the bifurcation of a center-type focus and the question remains unanswered for the predator-prey systems (2.1). It is well known that there are formula available to calculate the first Liapunov coefficient and the higher order Liapunov coefficients ( e.g. cf $[2,14]$ ), however, in general it is technically very challenging to draw conclusions from the formula directly due to its complexities. Pilyugin and Waltman $[15,16]$ develop a divergence criterion for a generic planar system and the applications were made successfully to the study of multiple limit cycles in the chemostat with variable yield and other planar systems.

In this paper, we are going to develop some criteria to determine the multiplicity and stability of the multiple focus for the general predator-prey system (2.1). Thanks to the magic of Liénard-type system, which has been playing an increasingly important role in current research on the existence and uniqueness of limit cycles for predator prey systems (cf [23] and reference therein). Hence it is not surprising that the equivalent form of (1.1), a Liénard-type system can be simpler in calculating the multiplicity for a focus.

Consider the general Liénard-type system

$$
\left\{\begin{array}{l}
\frac{d x}{d t}=\phi(y)-F(x), \\
\frac{d y}{d t}=-g(x),
\end{array}\right.
$$

where $x g(x)>0$ for $x \neq 0$. We first introduce a very useful lemma of [7] by Han.

Lemma 2.1. Suppose that $\phi(y), F(x)$ and $g(x)$ are $C^{\infty}$ smooth functions of its variable in a neighborhood of the origin, and

$$
\phi(0)=g(0)=F(0)=F^{\prime}(0)=0, \quad \phi^{\prime}(0)>0 \text { and } g^{\prime}(0)>0 .
$$

Let $G(x)=\int_{0}^{x} g(s) d s$. If there exists a $C^{\infty}$ smooth function $\alpha(x), \alpha(x)=-x+O\left(x^{2}\right)$, such that $G(\alpha(x)) \equiv G(x)$ and

$$
F(\alpha(x))-F(x)=\sum_{i \geq 1} B_{i} x^{i},
$$

then the equilibrium $(0,0)$ of (2.2) is a multiple focus of multiplicity $k$ if $B_{j}=0, j=$ $1,2, \cdots, 2 k$ and $B_{2 k+1} \neq 0$. And it is locally stable (unstable) if $B_{2 k+1}<0\left(B_{2 k+1}>0\right.$, respectively). 
The key to the proof of Lemma 2.1 is to transform (2.2) into

$$
\left\{\begin{array}{l}
\frac{d u}{d \tau}=v-K(v) F^{*}(u) \\
\frac{d v}{d \tau}=-u
\end{array}\right.
$$

by a $C^{\infty}$ transformation of variables $(x, y)$ near the origin and time $t$. Then let

$$
F_{e}(u)=\frac{1}{2}\left(F^{*}(u)+F^{*}(-u)\right), \quad F_{o}(u)=\frac{1}{2}\left(F^{*}(u)-F^{*}(-u)\right) .
$$

By the principle of symmetry, the orbits of the following system

$$
\left\{\begin{array}{l}
\frac{d u}{d \tau}=v-K(v) F_{e}(u) \\
\frac{d v}{d \tau}=-u
\end{array}\right.
$$

near the origin are symmetric with respect to the $v$-axis. In a small neighborhood of the origin, introducing the polar coordinates $u=r \cos \theta, v=r \sin \theta$ to (2.3) and (2.4), one can get two equations

$$
\frac{d r}{d \theta}=\frac{\cos \theta K(r \sin \theta) F^{*}(r \cos \theta)}{1-\sin \theta K(r \sin \theta) F^{*}(r \cos \theta) / r}
$$

and

$$
\frac{d r}{d \theta}=\frac{\cos \theta K(r \sin \theta) F_{e}(r \cos \theta)}{1-\sin \theta K(r \sin \theta) F_{e}(r \cos \theta) / r}
$$

respectively. According to the classical method of Lyapunov, we can define the displacement map $d\left(r_{0}\right)$ of $(2.5)$ as

$$
d\left(r_{0}\right)=r\left(-\frac{3 \pi}{2}, r_{0}\right)-r\left(\frac{\pi}{2}, r_{0}\right)
$$

for $0<r_{0} \ll 1$. From the technical analysis of $d\left(r_{0}\right)$, the conclusions of the lemma can be obtained. For more details on the proof of the lemma and applications, we refer the reader to [7] by Han.

We next utilize the technique developed in [23] to transform the general predator-prey system (2.1) into a Liénard-type system and use Lemma 2.1 to establish conditions to determine the multiplicity of the center-type focus. We focus our attention to system (2.1) with assumptions (A1)-(A5) in the open set $\Omega$.

Since $p(x)>0$, rescaling the time $t$ of system (2.1) by

$$
\tau=\int_{0}^{t} p(x(s)) d s
$$

we obtain

$$
\left\{\begin{aligned}
\frac{d x}{d \tau} & =\frac{a(x)}{p(x)}-b(y), \\
\frac{d y}{d \tau} & =c(y) \frac{q(x)}{p(x)}
\end{aligned}\right.
$$


System (2.8) has an equilibrium at $\left(x_{0}, y_{0}\right)$. Let

$$
x=-u+x_{0}, \quad y=h(v)+y_{0},
$$

we translate the equilibrium $\left(x_{0}, y_{0}\right)$ of system $(2.8)$ to the origin, where $h(v)$ is a solution of the following initial problem

$$
\frac{d h(v)}{d v}=c\left(h(v)+y_{0}\right), \quad h(0)=0 .
$$

Since $c^{\prime}(y)>0, h(v)$, the solution to the initial value problem $(2.10)$ exists for $v>0$ and it is unique. Thereby the inverse function of $h(v)$ exists. Let us denote the inverse function by $h^{-1}$. Thus, there exists an inverse transformation of (2.9) such that system (2.8) in $\Omega$ can be transformed to

$$
\left\{\begin{array}{l}
\frac{d u}{d \tau}=\left[b\left(h(v)+y_{0}\right)-b\left(y_{0}\right)\right]-\left[\frac{a\left(-u+x_{0}\right)}{p\left(-u+x_{0}\right)}-b\left(y_{0}\right)\right] \\
\frac{d v}{d \tau}=-\left[-\frac{q\left(-u+x_{0}\right)}{p\left(-u+x_{0}\right)}\right]
\end{array}\right.
$$

in the neighborhood of the origin. If we define

$$
\begin{aligned}
\phi(v) & =b\left(h(v)+y_{0}\right)-b\left(y_{0}\right), \\
F(u) & =\frac{a\left(-u+x_{0}\right)}{p\left(-u+x_{0}\right)}-b\left(y_{0}\right), \\
g(u) & =-\frac{q\left(-u+x_{0}\right)}{p\left(-u+x_{0}\right)},
\end{aligned}
$$

then system (2.11) becomes

$$
\left\{\begin{array}{l}
\frac{d u}{d \tau}=\phi(v)-F(u) \\
\frac{d v}{d \tau}=-g(u)
\end{array}\right.
$$

System (2.12) is a Liénard-type system in the neighborhood of the origin, and it can be observed that

$$
\phi(0)=g(0)=F(0)=F^{\prime}(0)=0, \quad \phi^{\prime}(0)>0 \text { and } g^{\prime}(0)>0 .
$$

Applying Lemma 2.1 to system (2.12), we obtain

Theorem 2.2. Assume that (A1) to (A5) hold for system (2.1). Suppose b(y) and $c(y)$ are $C^{\infty}$ functions of $y$ in a neighborhood of $y_{0}$, and $a(x) / p(x)$ and $q(x) / p(x)$ are $C^{\infty}$ functions of $x$ in a neighborhood of $x_{0}$. Let

$$
G(x)=\int_{0}^{x}-\frac{q\left(-u+x_{0}\right)}{p\left(-u+x_{0}\right)} d u .
$$


If there exists a $C^{\infty}$ function $\alpha(x), \alpha(x)=-x+O\left(x^{2}\right)$ such that $G(\alpha(x)) \equiv G(x)$ and

$$
F(\alpha(x))-F(x)=\frac{a\left(-\alpha(x)+x_{0}\right)}{p\left(-\alpha(x)+x_{0}\right)}-\frac{a\left(-x+x_{0}\right)}{p\left(-x+x_{0}\right)}=\sum_{i \geq 1} B_{i} x^{i},
$$

and if $B_{j}=0, j=1,2, \cdots, 2 k$ and $B_{2 k+1} \neq 0$, then the equilibrium $\left(x_{0}, y_{0}\right)$ of (2.1) is a multiple focus of multiplicity $k$, which is locally stable (unstable) if $B_{2 k+1}<0\left(B_{2 k+1}>0\right.$, respectively).

\section{Multiple focus and degenerate Hopf bifurcation}

Now we consider system (1.2). From the work of $[18,4]$ to $[19]$ and [26] and the references therein, there have been extensive studies of various bifurcations for system (1.2). It follows from [26] that system (1.2) undergoes bifurcations include saddle-node bifurcation, Hopf bifurcation(s), homoclinic bifurcation and saddle node bifurcation of limit cycles. Note that system (1.2) with the functional response (1.3) involves an extra parameter $b$. As indicated in [26] that by varying this parameter $b$, one can connect all the bifurcation branches to the organizing center, which is a Bogdanov-Takens bifurcation of codimension three. Although the bifurcation study for (1.2) in [26] is almost exhaustive, yet the codimension of the degenerate Hopf bifurcation was left untouched and the multiplicity of the multiple focus is unknown.

It follows from the discussion in [26] that by rescaling the state variables and time, one can eliminate 3 parameters from the system (1.2). For the purpose of combining the results from [19] and [26] regarding the bifurcations, here we eliminate $a, m$ and $c$ by using

$$
\begin{aligned}
& (t, x, y) \longrightarrow\left(\frac{\sqrt{a}}{m c} t, \frac{1}{\sqrt{a}} x, \frac{c}{\sqrt{a}} y\right) \\
& (r, K, b, d) \longrightarrow\left(\frac{m c}{\sqrt{a}} r, \frac{1}{\sqrt{a}} K, \sqrt{a} b, \frac{1}{\sqrt{a}} d\right) .
\end{aligned}
$$

Then system (1.2) with the functional response (1.3) becomes

$$
\left\{\begin{array}{l}
\dot{x}=r x\left(1-\frac{x}{K}\right)-\frac{x y}{x^{2}+b x+1} \\
\dot{y}=y\left(-d+\frac{x}{x^{2}+b x+1}\right) .
\end{array}\right.
$$

System (3.2) involves four parameters $b, d, K$ and $r$, where $r>0$ is not a bifurcation parameter for the equilibria. It follows from the results in [26] that we need three parameters to unfold the Bogdanov-Takens bifurcation of codimension three, hence it is natural to describe the degenerate Hopf bifurcation curve in the parameter space $(b, d, K)$. We start first by summarizing the results from [26] regarding the Hopf bifurcations for system (3.2), then we finish the diagram by applying Theorem 2.2 to determine the multiplicity of the multiple focus and complete the diagram of Hopf bifurcation.

In [26], the geometry of the isoclines plays an important role in understanding both the equilibria and their bifurcations. Denote the prey isoline of (3.2) as (still use the 
notation in [26] but with a hat)

$$
\hat{F}(x)=r\left(1-\frac{x}{K}\right)\left(x^{2}+b x+1\right) .
$$

It follows from the linear stability analysis in [26] that besides the equilibria at the origin and $(K, 0)$, if $d<\frac{1}{b+2}$, system (3.2) may have up to two possible positive equilibria $\left(x_{i}, y_{i}=\hat{F}\left(x_{i}\right)\right)\left(i=1,2, x_{1}<x_{2}\right)$, if $x_{1}$ and $x_{2}$ are positive and smaller than $K$, where

$$
\begin{aligned}
& x_{1}=\frac{1-b d-\sqrt{\left(b^{2}-4\right) d^{2}-2 b d+1}}{2 d}, \\
& x_{2}=\frac{1-b d+\sqrt{\left(b^{2}-4\right) d^{2}-2 b d+1}}{2 d} .
\end{aligned}
$$

Note that $x_{1}$ and $x_{2}$ are the real roots of the quadratic equation

$$
\hat{g}(x)=d x^{2}-(1-b d) x+d=0
$$

and we also have

$$
x_{1}+x_{2}=\frac{1-b d}{d}, \quad x_{1} x_{2}=1 .
$$

The equilibrium $\left(x_{2}, y_{2}\right)$ is always a hyperbolic saddle if $0<x_{1}<x_{2}<K$. The stability of $\left(x_{1}, y_{1}\right)$ as a hyperbolic focus (or node) can be found in [26]. Here we are only interested in the equilibrium $\left(x_{1}, y_{1}\right)$ and related Hopf bifurcations. Recall from [26] that $\left(x_{1}, y_{1}\right)$ may undergo a Hopf bifurcation if $\hat{F}^{\prime}\left(x_{1}\right)=0$. The prey isocline can have two humps $\left(H_{m}, \hat{F}\left(H_{m}\right)\right)$ and $\left(H_{M}, \hat{F}\left(H_{M}\right)\right)$, where

$$
\begin{aligned}
& H_{m}=\frac{1}{3 a}\left[a K-b-\sqrt{a^{2} K^{2}+a b K+b^{2}-3 a}\right], \\
& H_{M}=\frac{1}{3 a}\left[a K-b+\sqrt{a^{2} K^{2}+a b K+b^{2}-3 a}\right] .
\end{aligned}
$$

As shown in Fig. 1, when a Hopf bifurcation occurs, it occurs at either the left hump $\left(H_{m}, \hat{F}\left(H_{m}\right)\right)$ or the right hump $\left(H_{M}, \hat{F}\left(H_{M}\right)\right)$. By (3.6) we know that $x_{1} \leq 1$. Hence for the Hopf bifurcation to occur at a hump of $F(x)$, the hump cannot be at the right of the vertical line $x=1$ (Corollary 4.2 in [26]).

Since if the Hopf bifurcation occurs, it occurs at one of the humps of $\hat{F}(x)$, or where $\hat{F}^{\prime}(x)=0$. Hence the Hopf bifurcation can only occur at either $x_{1}=H_{m}$ or $x_{1}=H_{M}$. Note that we can also solve $\hat{F}^{\prime}\left(x_{1}\right)=0$ in terms of $K$, then when the Hopf bifurcation occurs at $x=x_{1}$, we should also have

$$
K=\frac{1+2 b x_{1}+3 x_{1}^{2}}{b+2 x_{1}} .
$$

Substituting $x_{1}$ into (3.8) or eliminating $x$ from $\hat{g}(x)=0$ and $\hat{F}^{\prime}(x)=0$, one gets the Hopf bifurcation surface (Eq. (4.2) in [26]) in the parameter space $(b, K, d)$ :

$$
\Sigma_{H}:\left(4-b^{2}\right)\left(K^{2}+b K+1\right) d^{2}+2\left(b K^{2}+2\left(b^{2}-2\right) K+b\right) d+3(1-b K)=0 .
$$




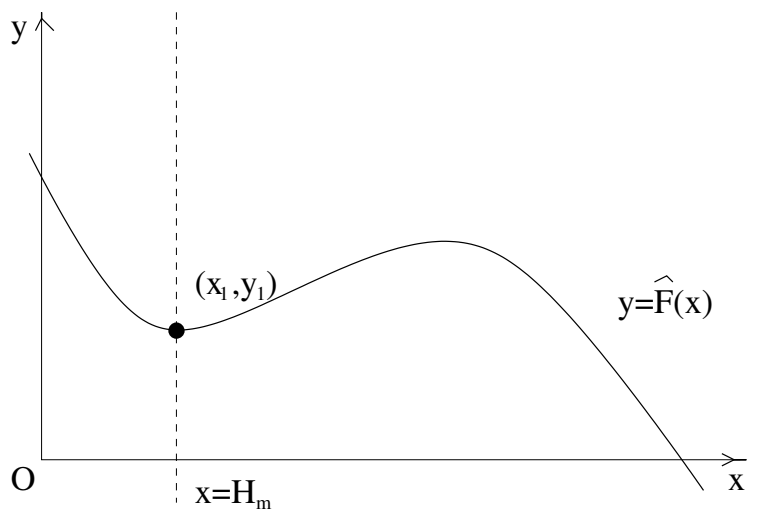

(a) At the left hump, the Hopf bifurcation can be both subcritical and supercritical

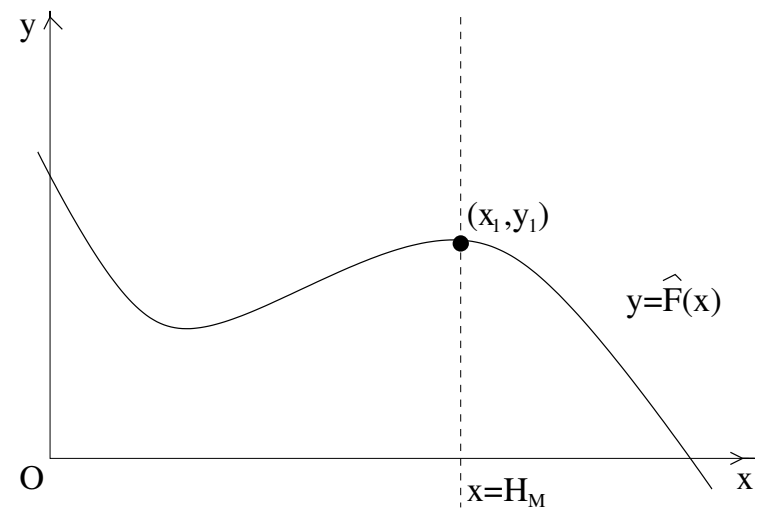

(b) At the right hump, the Hopf bifurcation is always supercritical

Figure 1: Hopf bifurcation occurs at the humps of the prey isocline

Solving for $d$ we obtain

$$
d_{ \pm}(b, K)=\frac{-\left(b K^{2}+2\left(b^{2}-2\right) K+b\right) \pm(2+b K) \sqrt{K^{2}+b K+b^{2}-3}}{\left(4-b^{2}\right)\left(K^{2}+b K+1\right)} .
$$

Hence for any $(b, K)$ such that $\hat{F}(x)$ has an hump to the left of the vertical line $x=1$, if $d=d_{-}$or $d=d_{+}$, the Hopf bifurcation may occur. By using the formula given by Wolkowicz [21], the first Lypunov coefficient was calculated in [26], and

$$
\sigma(x)=-\frac{p(x) \hat{F}^{\prime \prime}(x) p^{\prime \prime}(x)}{p^{\prime}(x)}+p(x) \hat{F}^{\prime \prime \prime}(x)+2 p^{\prime}(x) \hat{F}^{\prime \prime}(x) .
$$

It was then proved that for the Hopf bifurcation occurs at the right hump, i.e., $x_{1}=H_{M}$, we have $\sigma\left(H_{M}\right)<0$. Hence if the Hopf bifurcation occurs at the right hump, it is always supercritical. For the Hopf bifurcation occurs at the left hump when $d=d_{-}$, it can be both subcritical and supercritical.

Plugging $x_{1}$ from (3.4) into $\sigma\left(x_{1}\right)=0$, in [26] a degenerate Hopf bifurcation curve was defined. Let

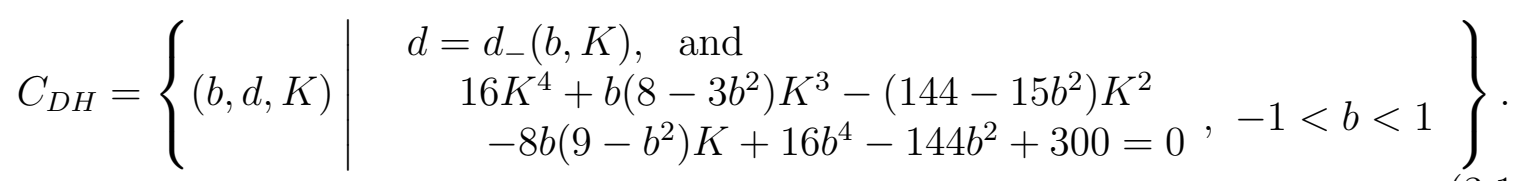

Then it follows from the results in [26] that $C_{D H}$ is a curve segment in the Hopf bifurcation surface $\Sigma_{H}$, and the projection of the curve onto the $(b, K)$ plane was plotted in Fig. 2), it is a curve segment with the endpoints at $P(-1,2)$ and $Q(1,1)$. In next Proposition, we summarize the result regarding the Hopf bifurcation(s) of codimension one from [26].

Proposition 3.1. If the Hopf bifurcation occurs at the right hump when $d=d_{+}$, it is always supercritical. For the Hopf bifurcation occurs at the left hump when $d=d_{-}$, as 


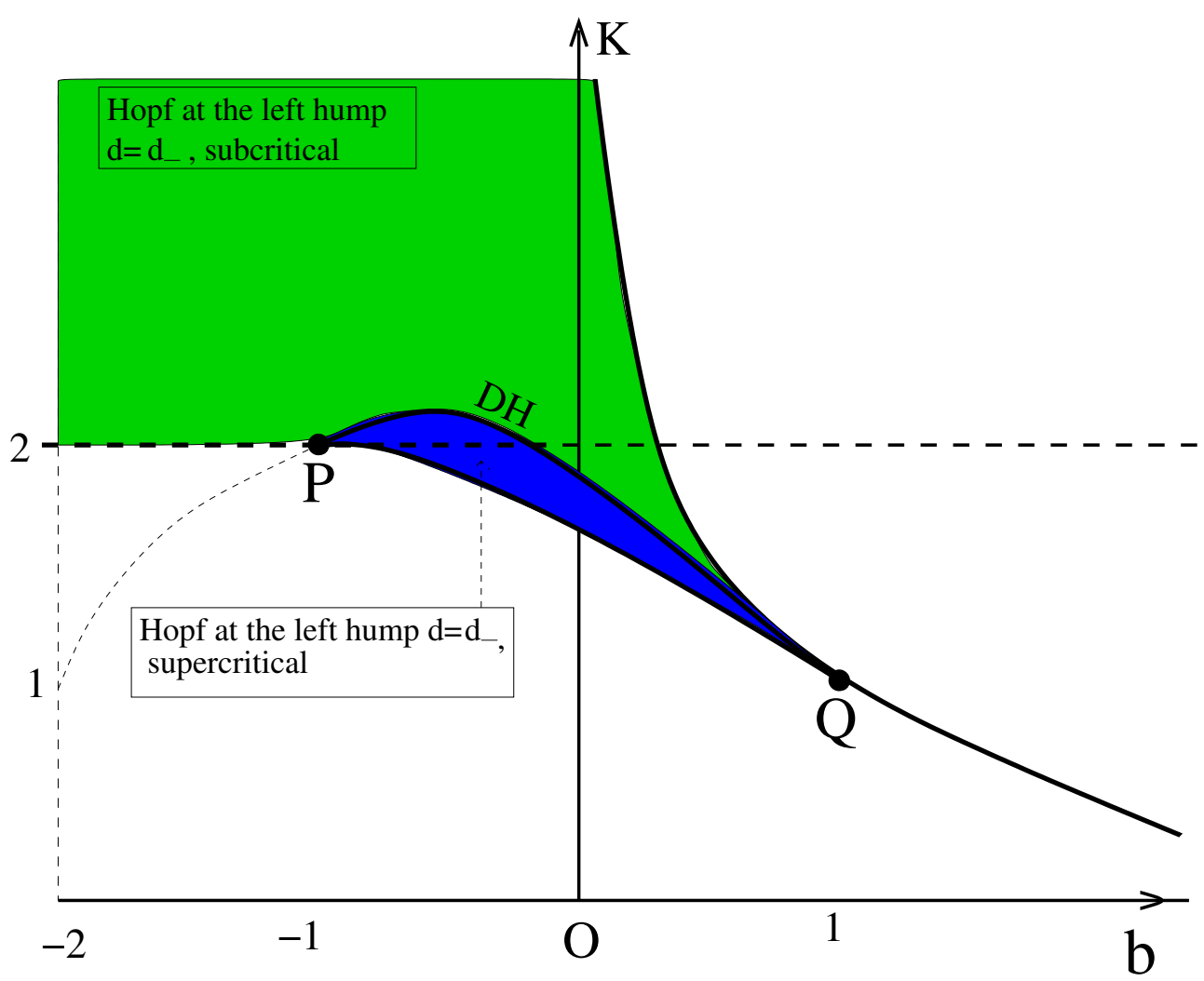

Figure 2: Hopf bifurcation occurs at the left hump when $d=d_{-}(b, K)$. The curve segment $D H$, the projection of the curve segment $C_{D H}$ into the $b K$ plane is plotted. It separates the colored region into two: for $(b, k)$ in the blue region and $d=d_{-}$, the Hopf bifurcation at the left hump is supercritical; for $(b, k)$ in the green region and $d=d_{-}$, the Hopf bifurcation at the left hump is subcritical, [26]. Here $C_{1}: K=\frac{1}{2}\left[\sqrt{3\left(4-b^{2}\right)}-b\right]$, $-2 \leq b \leq 1 ; C_{2}: K=\frac{1}{b}, b>0$. For $(b, K)$ below $C_{1}, \hat{F}(x)$ does not have any humps, to the right of $C_{2}$, there is only the right hump in the positive cone. Above the line $K=2$, only one hump is to the left of $x=1$.

shown in Fig. 2, it is subcritical if $(b, K)$ is above $D H$ (red region), it is supercritical if $(b, K)$ is below $D H$ (blue). For $(b, K) \in D H$ and $d=d_{-}$, a degenerate Hopf bifurcation occurs.

By an easy computation from (3.8) and (3.4), when a Hopf occurs, we have

$$
\begin{aligned}
K & =K_{0}(b, d)=\frac{2-\sqrt{(1-b d)^{2}-4 d^{2}}}{1-\sqrt{(1-b d)^{2}-4 d^{2}}} x_{1} \\
& =\left(1+\frac{1}{1-\sqrt{(1-b d)^{2}-4 d^{2}}}\right) x_{1} .
\end{aligned}
$$

Since the equilibrium $\left(x_{1}, y_{1}\right)$ is isolate in the interior of the first quadrant, there exists a neighborhood $\Omega$ of $\left(x_{1}, y_{1}\right)$ such that system (3.2) has no other equilibria in $\Omega$ except 
$\left(x_{1}, y_{1}\right)$. Next we investigate the multiplicity of the multiple focus $\left(x_{1}, y_{1}\right)$ of system (3.2) when $(b, d, K) \in C_{D H}$ or equivalently when $K=K_{0}(b, d)$ and $d=d_{-}$. Note that in manipulating the expressions for conclusion, the relation (3.6) of $x_{1}$ and $x_{2}$ with the coefficients of the quadratic equation $\hat{g}(x)=0$ will be repeatedly used.

Taking $h(v)=y_{1}\left(e^{v}-1\right)$, we transform system (3.2) in $\Omega$ to a Liénard-type of system by transformations $(2.7)$ and $(2.9)$ when $K=K_{0}(b, d)$ and $d=d_{-}$. For simplicity, we still denote $u, v$ and $\tau$ by $x, y$ and $t$, respectively. Then system (1.2) can be written as

$$
\left\{\begin{array}{l}
\frac{d x}{d t}=\phi(y)-F(x), \\
\frac{d y}{d t}=-g(x)
\end{array}\right.
$$

where

$$
\begin{aligned}
\phi(y) & =y_{1}\left(e^{y}-1\right), \\
F(x) & =\hat{F}\left(-x+x_{1}\right)-y_{1}=r\left(\left(1-\frac{b+3 x_{1}}{K_{0}}\right) x^{2}+\frac{x^{3}}{K_{0}}\right), \\
g(x) & =\frac{d}{p\left(-x+x_{1}\right)}-1=\frac{d x\left(x-x_{1}+x_{2}\right)}{x_{1}-x},
\end{aligned}
$$

where $(x, y) \in \Omega_{1}$, a neighborhood of the origin which comes from $\Omega$ under the transformation $(2.9)$.

It is clear that $\phi(y), F(x)$ and $g(x)$ are $C^{\infty}$ smooth functions in $\Omega_{1}$, and

- $\phi(0)=g(0)=F(0)=F^{\prime}(0)=0$,

- $\phi^{\prime}(0)=y_{1}>0$,

- $g^{\prime}(0)=\frac{p^{\prime}\left(x_{1}\right)}{p^{2}\left(x_{1}\right)}=d\left(\frac{x_{2}}{x_{1}}-1\right)>0$ and

$$
G(x)=\int_{0}^{x}\left[\frac{d}{p\left(-u+x_{1}\right)}-1\right] d u=-d\left(\frac{1}{2} x^{2}+x_{2} x+x_{1} x_{2} \ln \frac{x_{1}-x}{x_{1}}\right) .
$$

A straightforward computation can verify that there exists a $C^{\infty}$ smooth function

$$
\alpha(x)=-x+a_{2} x^{2}+a_{3} x^{3}+a_{4} x^{4}+O\left(x^{5}\right),
$$

such that $G(\alpha(x)) \equiv G(x)$, where

$$
\begin{aligned}
& a_{2}=-\frac{G^{\prime \prime \prime}(0)}{3 G^{\prime \prime}(0)}=-\frac{2\left(p^{\prime}\left(x_{1}\right)\right)^{2}-p\left(x_{1}\right) p^{\prime \prime}\left(x_{1}\right)}{3 p\left(x_{1}\right) p^{\prime}\left(x_{1}\right)}=-\frac{2 x_{2}}{3 x_{1}\left(x_{2}-x_{1}\right)} \\
& a_{3}=-a_{2}^{2} \\
& a_{4}=a_{2}\left(2 a_{2}^{2}+\frac{3 a_{2}}{2 x_{1}}+\frac{3}{5 x_{1}^{2}}\right) .
\end{aligned}
$$

Performing a Taylor expansion of function $F(\alpha(x))-F(x)$ at $x=0$, we obtain

$$
F_{2}(\alpha(x))-F_{2}(x)=r\left(A_{3} x^{3}+A_{4} x^{4}+A_{5} x^{5}+O\left(x^{6}\right)\right),
$$


where

$$
\begin{aligned}
& A_{3}=\frac{1}{3}\left[F^{\prime \prime \prime}\left(x_{1}\right)-3 a_{2} F^{\prime \prime}\left(x_{1}\right)\right]=-2 a_{2}\left(1-\frac{b+3 x_{1}}{K_{0}}\right)-\frac{2}{K_{0}}, \\
& A_{4}=\left(1-\frac{b+3 x_{1}}{K_{0}}\right)\left(a_{2}^{2}-2 a_{3}\right)+\frac{3 a_{2}}{K_{0}} \\
& A_{5}=\left(1-\frac{b+3 x_{1}}{K_{0}}\right)\left(2 a_{2} a_{3}-2 a_{4}\right)+\frac{3 a_{3}-3 a_{2}^{2}}{K_{0}} .
\end{aligned}
$$

It follows from (3.11) and (3.17) that

$$
\begin{aligned}
A_{3} & =\frac{1}{3 p\left(x_{1}\right)}\left[p F^{\prime \prime \prime}\left(x_{1}\right)+\frac{2\left(p^{\prime}\left(x_{1}\right)\right)^{2}-p\left(x_{1}\right) p^{\prime \prime}\left(x_{1}\right)}{p^{\prime}\left(x_{1}\right)} F^{\prime \prime}\left(x_{1}\right)\right] \\
& =\frac{1}{3 p\left(x_{1}\right)} \sigma\left(x_{1}\right) .
\end{aligned}
$$

Hence if $\sigma\left(x_{1}\right) \neq 0, A_{3} \neq 0$, the equilibrium $(0,0)$ of system (3.14) is a multiple focus of multiplicity one.

Along the curve $C_{D H}$ where $\sigma\left(x_{1}\right)=0$, we have $A_{3}=0$. Then the equilibrium $(0,0)$ is a multiple focus of multiplicity at least two and we have to calculate $A_{4}, A_{5} \ldots$ in order to determine the multiplicity of equilibrium $(0,0)$. For the purpose of identifying the sign for $A_{5}$, we need the fact that along $D H, A_{3}=0$ and it is equivalent to

$$
x_{2}=\frac{\left(3 b+6 x_{1}\right) x_{1}^{2}}{12 x_{1}^{2}+9 b x_{1}+2\left(b^{2}-1\right)} .
$$

Assuming that $A_{3}=0$, and using the expressions of $a_{i}(i=2,3,4)$ and condition (3.20), we compute

$$
\begin{aligned}
A_{4} & =-\frac{1}{a_{2} K_{0}}\left(a_{2}^{2}-2 a_{3}\right)+\frac{3 a_{2}}{K_{0}}=0, \\
A_{5} & =-\frac{1}{a_{2} K_{0}}\left(2 a_{2} a_{3}-2 a_{4}\right)+\frac{3 a_{3}-3 a_{2}^{2}}{K_{0}} \\
& =\frac{3}{5 K_{0} x_{1}}\left(5 a_{2}+\frac{2}{x_{1}}\right) \\
& =-\frac{2\left(3 x_{1}+2 x_{2}\right)}{5 K_{0} x_{1}^{2}\left(x_{2}-x_{1}\right)}<0 .
\end{aligned}
$$

Thus, equilibrium $(0,0)$ is a stable multiple focus of multiplicity two by Theorem 2.2 . Summarizing above arguments, we obtain

Theorem 3.2. For $(b, d, K) \in C_{D H}$, or equivalently, if $(b, K) \in D H$ with $-1<b<1$ and $d=d_{-}$, the origin of system (3.14) (i.e. equilibrium $\left(x_{1}, y_{1}\right)$ of system (3.2)) is a multiple focus of multiplicity two, which is locally asymptotically stable.

From Theorem 3.2, we immediately have the result regarding the degenerate Hopf bifurcation.

Theorem 3.3. For $(b, d, K) \in C_{D H}$, system (3.2) undergoes a Hopf bifurcation of codimension two, and the diagram for the Hopf bifurcation occurring at the left hump, Fig. 2, is complete. 
Remark 3.4. It follows from the expression (3.21) that $A_{5}$ goes to infinity if $x_{1}$ and $x_{2}$ are getting close enough. This is consistent with the fact that the degenerate Hopf curve $C_{D H}$ is connected to the Bogdanov-Takens bifurcation point of codimension three, where $x_{1}$ and $x_{2}$ coincide together at the infection point of the prey isocline $y=\hat{F}(x)$.

Using standard Hopf bifurcation theorem in [2] and [14], we can see that system (3.2) undergoes a supercritical Hopf bifurcation of codimension 1 and a subcritical Hopf bifurcation of codimension 1 in succession when $d=d_{-}$and as $(b, K)$ moves from below to the above of the curve $D H$. Therefore, exactly two limit cycles may appear.

To discuss the exact number of limit cycles of system (3.2) via Hopf bifurcations, by restricting to the case $b=0$, we can prove that system (3.2) has no limit cycles if system (3.2) has a multiple focus of multiplicity two. More precisely, we have the following theorem.

Theorem 3.5. Consider system (3.2) with $b=0$. Assume that $0<x_{1}<K \leq K_{0}$ and $\sqrt{\frac{3}{18+2 \sqrt{6}}} \leq d<\frac{\sqrt{3}}{4}$ then the equilibrium $\left(x_{1}, y_{1}\right)$ of system (3.2) is globally stable in the interior of $R_{+}^{2}$ (Note that $\left(x_{1}, y_{1}\right)$ of system (3.2) is a multiple focus of multiplicity two when $b=0, K=K_{0}$ and $\left.d=\sqrt{\frac{3}{18+2 \sqrt{6}}}\right)$.

Proof. It is clear that system (3.2) has a unique positive equilibrium $\left(x_{1}, y_{1}\right)$ if $b=0$, $0<x_{1}<K \leq K_{0}$ and $\sqrt{\frac{3}{18+2 \sqrt{6}}} \leq d<\frac{\sqrt{3}}{4}$. Since the solutions of (3.2) in the interior of $R_{+}^{2}$ are positive and eventually bounded (i.e. there exists a positive number $T$ such that $0<x(t)<K$ for $t>T)$, we claim that the unique positive equilibrium $\left(x_{1}, y_{1}\right)$ of system (3.2) is globally stable in the interior of $R_{+}^{2}$. We prove the result by showing that system (3.2) has no closed orbits in the domain $\Omega_{2}$, where

$$
\Omega_{2}=\{(x, y): 0<x<K, \quad 0<y<+\infty\}
$$

Taking $h(v)=y_{1}\left(e^{v}-1\right)$, we transform system (3.2) in $\Omega_{2}$ to the following Liénardtype of system by transformations (2.7) and (2.9) when $b=0,0<x_{1}<K \leq K_{0}$ and $\sqrt{\frac{3}{18+2 \sqrt{6}}} \leq d<\frac{\sqrt{3}}{4}$ ( we still denote $u, v$ and $\tau$ by $x, y$ and $t$, respectively)

$$
\begin{aligned}
& \frac{d x}{d t}=\phi_{1}(y)-F_{1}(x), \\
& \frac{d y}{d t}=-g_{1}(x)
\end{aligned}
$$

in the domain $\Omega_{3}$, where $\phi_{1}(y)=y_{1}\left(e^{y}-1\right), F_{1}(x)=\frac{r x}{K}\left(x^{2}+\left(K-3 x_{1}\right) x+x_{1}\left(x_{2}+3 x_{1}-K\right)\right)$, $g_{1}(x)=\frac{d x\left(x-x_{1}+x_{2}\right)}{x_{1}-x}$, and

$$
\Omega_{3}=\left\{(x, y): x_{1}-K<x<x_{1},-\infty<y<+\infty\right\} .
$$

Note that the problem of existence of closed orbits of system (3.2) in the domain $\Omega_{2}$ is equivalent to that of system (3.22) in the domain $\Omega_{3}$. 
By the Filippov transformation, we can see that system (3.22) has no closed orbits if $\frac{f_{1}(x)}{g_{1}(x)} \leq \frac{f_{1}(u)}{g_{1}(u)}$ holds for any $(u, x)$ satisfying $G_{1}(x)=G_{1}(u)$ with $x_{1}-K<u<0$ and $0<x<x_{1}$ (cf Theorem 2.5 in [23]), where $G_{1}(x)=\int_{0}^{x} g_{1}(s) d s$, and

$$
f_{1}(x) \stackrel{\text { def }}{=} F_{1}^{\prime}(x)=\frac{r}{k}\left(3 x^{2}+2\left(K-3 x_{1}\right) x+x_{1}\left(x_{2}+3 x_{1}-K\right)\right) .
$$

Next we claim that $u+x<0$ if $G_{1}(u)=G_{1}(x)$ with $x_{1}-K<u<0$ and $0<x<x_{1}$. In fact, if

$$
G_{1}(u)=G_{1}(x)
$$

as $x_{1}-K<u<0$ and $0<x<x_{1}$, then

$$
\frac{1}{2}(x-u)(x+u)+x_{2}(x-u)-x_{1} x_{2} \ln \frac{x_{1}-u}{x_{1}-x}=0 .
$$

Note that $2 \frac{x-y}{x+y} \leq \ln \frac{x}{y}$ as $0<y \leq x$, and the equality holds if and only if $x=y$. Hence, we have

$$
(x+u)\left(x_{1}-x_{2}-\frac{1}{2}(x+u)\right)>0,
$$

which implies that $2\left(x_{1}-x_{2}\right)<u+x<0$.

As $G_{1}(u)=G_{1}(x)$ with $x_{1}-K<u<0$ and $0<x<x_{1}$, we consider

$$
\begin{aligned}
\frac{f_{1}(x)}{g_{1}(x)}-\frac{f_{1}(u)}{g_{1}(u)}= & \frac{r}{K d x u\left(x-x_{1}+x_{2}\right)\left(u-x_{1}+x_{2}\right)}\left(\left[3 x^{2}+2\left(K-3 x_{1}\right) x\right.\right. \\
& \left.+x_{1}\left(x_{2}+3 x_{1}-2 K\right)\right]\left(x_{1}-x\right) u\left(u-x_{1}+x_{2}\right)-\left[3 u^{2}+2\left(K-3 x_{1}\right) u\right. \\
& \left.\left.+x_{1}\left(x_{2}+3 x_{1}-2 K\right)\right]\left(x_{1}-u\right) x\left(x-x_{1}+x_{2}\right)\right) \\
= & \frac{r(x-u)}{K d x u\left(x-x_{1}+x_{2}\right)\left(u-x_{1}+x_{2}\right)}\left(-3 u^{2} x^{2}-3\left(x_{2}-x_{1}\right) x u(x+u)\right. \\
& -\left(2 K x_{2}+2 K x_{1}-10 x_{1} x_{2}\right) x u+\left(2 K-x_{2}-3 x_{1}\right) x_{1}^{2}(x+u) \\
& \left.+x_{1}^{2}\left(2 K-x_{2}-3 x_{1}\right)\left(x_{2}-x_{1}\right)\right) \\
= & \frac{r(x-u)}{K d x u\left(x-x_{1}+x_{2}\right)\left(u-x_{1}+x_{2}\right)}\left(-3 u^{2} x^{2}-3\left(x_{2}-x_{1}\right) x u(x+u)\right. \\
& \left.+\left(10 x_{1} x_{2}-2 K x_{2}-2 K x_{1}\right) x u-\left(x_{2}+3 x_{1}-2 K\right) x_{1}^{2}\left(x+u+x_{2}-x_{1}\right)\right) .
\end{aligned}
$$

Because $\sqrt{\frac{3}{18+2 \sqrt{6}}} \leq d<\frac{\sqrt{3}}{4}$ and $0<x_{1}<K \leq K_{0}=\left(3 x_{1}+x_{2}\right) / 2,10 x_{1} x_{2}-2 K x_{2}-$ $2 K x_{1} \geq 0$ and $x_{2}+3 x_{1}-2 K>0$. On the other hand, $x+u+x_{2}-x_{1}>x_{2}-K>0$. Therefore,

$$
\frac{f_{1}(x)}{g_{1}(x)}-\frac{f_{1}(u)}{g_{1}(u)}>0
$$

From above arguments and Theorem 2.5 in [23], we know that system (3.22) does not have any closed orbits in the range $\Omega_{3}$, which implies statement is true.

In Fig. 3, a numerical simulation was carried out by using Maple [20] to verify the existence of two limit cycles. For the simulation, we take $r=2$ and $b=0$, the other 


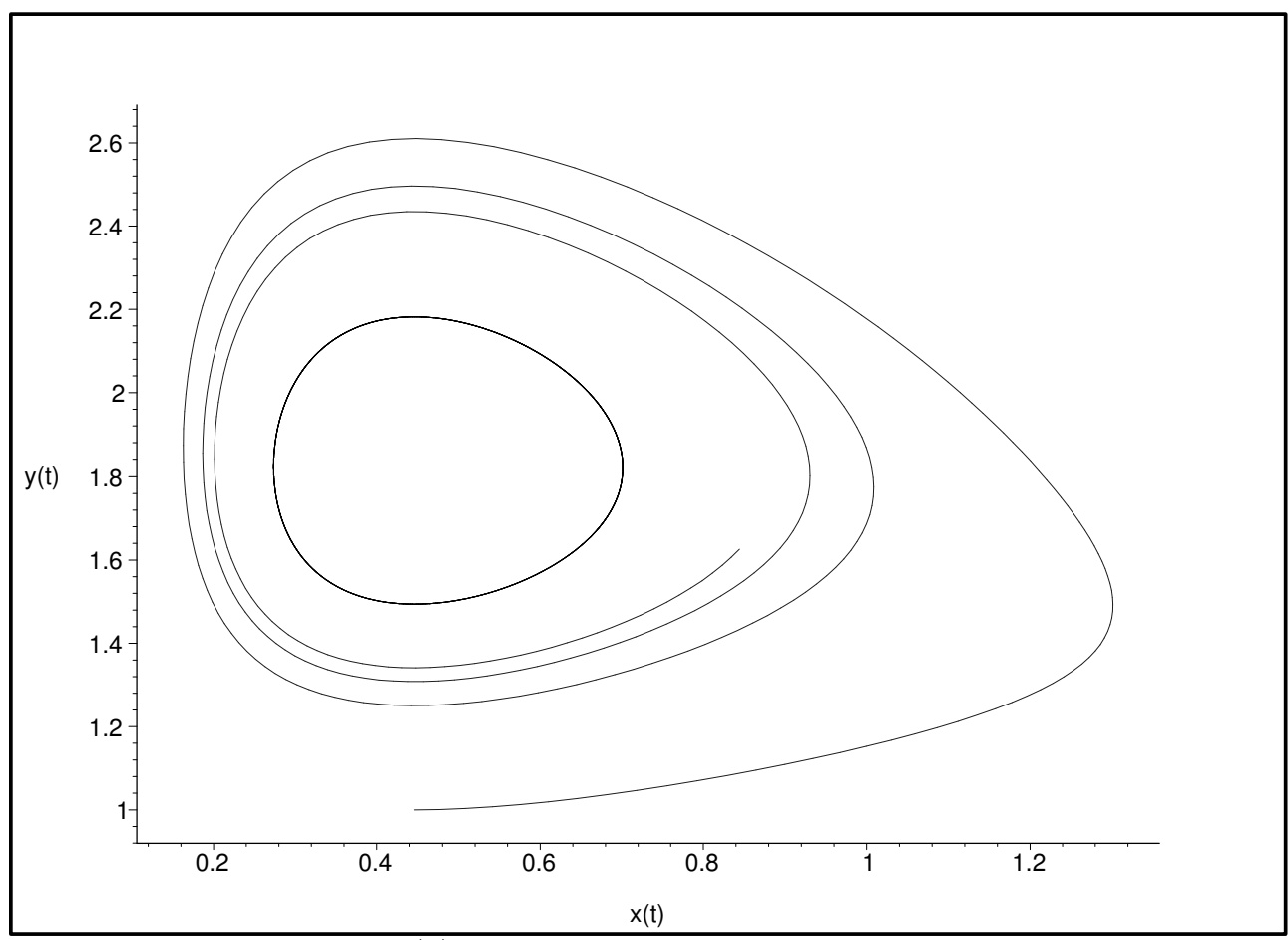

(a) The outer stable limit cycle

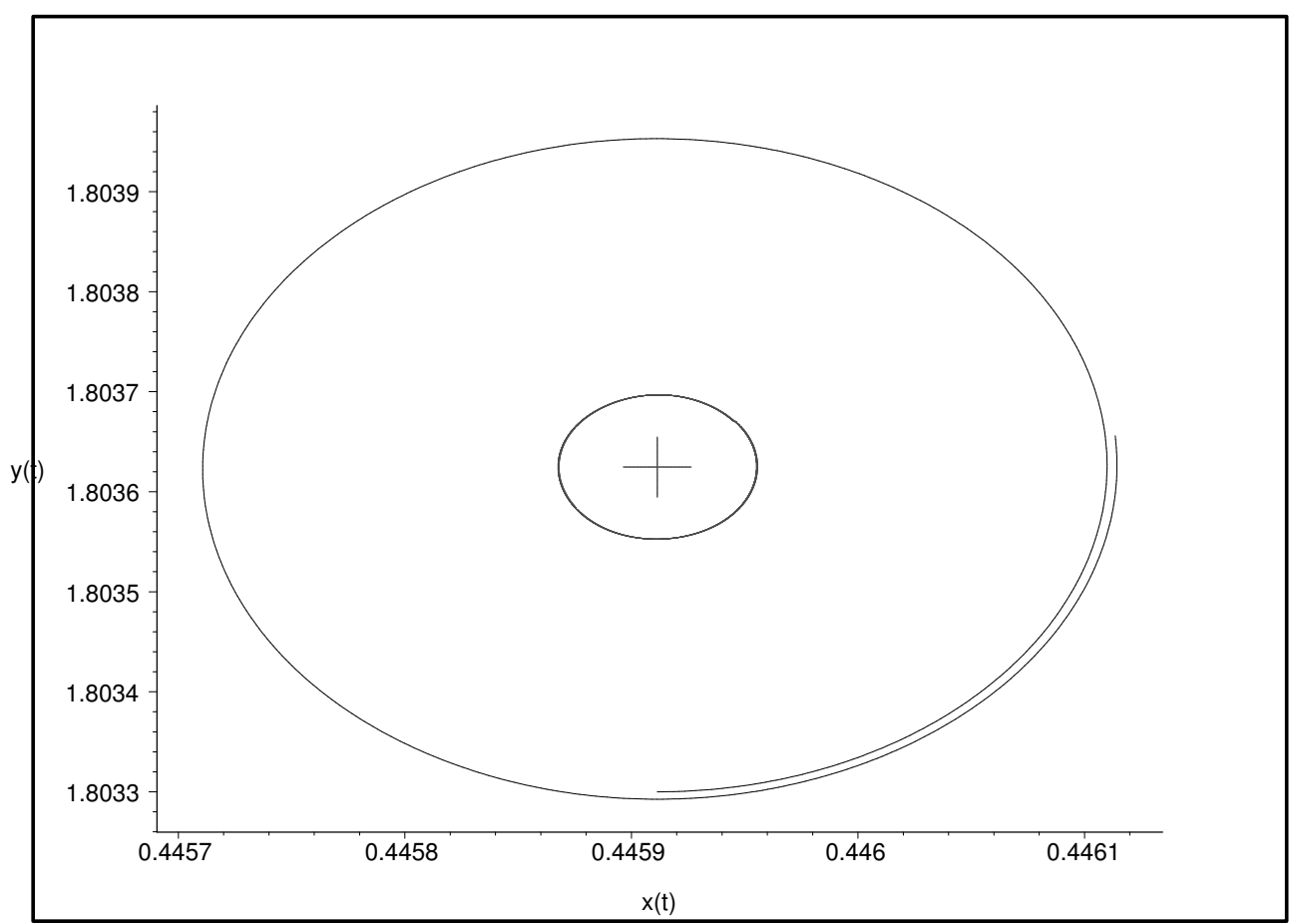

(b) The equilibrium $\left(x_{1}, y_{1}\right)$ is stable and the trajectory near by spirals outwards, which indicates the existence of an unstable limit cycle

Figure 3: Two limit cycles bifurcated from the multiple focus of multiplicity two. The figures were produced using MAPLE [20]. 


\begin{tabular}{|c|c|c|c|c|}
\hline & $d$ & $K$ & $x_{1}$ & $x_{2}$ \\
\hline Multiple focus & $\sqrt{\frac{3}{18+2 \sqrt{6}}}$ & $K_{0}=1.809766597$ & .4283729906 & 2.334414218 \\
\hline Perturbed & $\sqrt{\frac{3}{18+2 \sqrt{6}}+0.01}$ & $K_{0}=1.809766597+.01$ & .4459113886 & 2.242598026 \\
\hline
\end{tabular}

Table 1: Parameters value for the simulation to verify the existence of two limit cycles

parameters are given in Table 1, where system has a unique positive equilibrium which is a multiple focus of multiplicity two. We then perturb the parameters $d$ and $K$, the new perturbed system has two limit cycles as illustrated in Fig. 3 (a) and (b).

Remark 3.6. In [26], the saddle-node bifurcation of limit cycles were studied and the existence of two limit cycles were proved. In fact, it follows from the results in [26] and Theorem 3.2 and 3.4, that the bifurcation of the multiple focus of multiplicity two produces two limit cycles, and these two cycles can either disappear through the Hopf bifurcations or one Hopf bifurcation and one supercritical homoclinic bifurcation or disappear through the saddle-node bifurcation of limit cycles.

Remark 3.7. In [26] Theorem 6.22, for parameters $(b, K)$ in certain region near the curve segment $D H$, the bifurcation sequences were given but not complete. There we could not exclude the saddle-node bifurcations of limit cycles and supercritical homoclinic bifurcations. By the above Theorem 3.4 and 3.5, now we can conclude that the sequences given in Theorem 6.22 is complete for $(b, K)$ in the neighborhood of $b=0$ and near the curve segment DH.

\section{Discussion.}

The existence of limit cycles in predator-prey systems can be used to explain many real world oscillatory phenomena, such as the Canadian lynx-snowshoe hare 10-year cycles. However, the classical Lotka-Volterra predator-prey system does not exhibit periodic fluctuations since the positive equilibrium is globally stable. One can perturb the classical Lotka-Volterra predator-prey system to obtain a unique nonconstant periodic orbit (see Freedman [5]). The second method to modify the Lotka-Volterra predator-prey model so that it exhibits periodic solutions is to introduce functional response function. Various forms of functional response functions have been proposed. In general, most of these functional response functions are monotone and have self-saturation effect. The dynamics of the generalized Gause-type predator-prey systems with general monotonic functional response have been extensively studied and very well understood. Multiple limit cycles were observed in predator-prey systems for both the case when the predator death rate is linear [26] and nonlinear (Hofbauer and So [8], Kuang [9], Wrzosek [22], etc.). However, the existence of exactly two limit cycles in predator-prey systems has not been studied yet. 
Nonmonotonic functional response has been used to model the group defense phenomenon in population dynamics and the inhibition in microbial dynamics (Freedman and Wolkowicz [6], Mischaikow and Wolkowicz [12], Rosenzweig [17], and Wolkowicz [21]) and very rich and interesting dynamics have been observed in Gause-type predator-prey models with nonmonotonic functional response and only linear predator death rate $([18]$, [19] and [26]). However, it is difficult to discuss the degenerate Hopf bifurcation for these models since it is hard to determine the multiplicity of a multiple focus by usual methods in $[2,14]$. In this paper, we introduce a new method to solve the problem successfully. For the Gause-type predator prey model with nonmonotonic functional response as in (1.2), we have shown that it has a multiple focus with multiplicity exactly two, and there exist some parameter values for which system (1.2) with the functional response (1.3) has exactly two limit cycles. These results also extend the bifurcation analysis of (3.2) by Ruan and Xiao [19] and Zhu, Campbell and Wolkowicz [26].

It is natural that the predator prey interaction has the tendency or potential to produce periodic oscillations. Hence one always needs to study the multiplicity of multiple focus and Hopf bifurcations in such type of predator prey systems. The machinery developed in this paper can also be applied to the study of the multiplicity of multiple focus of other predator prey systems with linear death rate for the predator, even with harvesting.

Acknowledgements The authors are grateful to the anonymous referees and the handling editor for their valuable comments and suggestions. The authors also thank one referee for pointing us the reference [16].

\section{References}

[1] F. Albrecht, H. Gatzke And N. WAx, Stable limit cycles in pre-predator populations, Science 181 (1973), 1073-1074.

[2] A. Andronov, E. A. Leontovich, I. I. Gordon and A. G. Maier, Theory of Bifurcations of Dynamical Systems on a Plane, Israel Program for Scientific Translations, Jerusalem, 1971.

[3] S. N. Chow and J. K. Hale, Methods of Bifurcation Theory, Springer-Verlag, New York, 1982.

[4] A. D. Bazykin Nonlinear Dynamics of Interacting Populations. World Scientific Series on Nonlinear Science. Series A: Monographs and Treatises, 11. World Scientific Publishing Co., Inc., River Edge, NJ, 1998.

[5] H. I. Freedman, Deterministic Mathematical Models in Population Ecology, Marcel Dekker, New York, 1980.

[6] H. I. Freedman and G. S. K. Wolkowicz, Predator-prey systems with group defence: the paradox of enrichment revisited, Bull. Math. Biol. 48 (1986), 493-508. 
[7] M. HAn, Liapunov constants and Hopf cyclicity of Lienard systems, Ann. of Differential Equations 15 (1999), 113-126.

Available through: http://www.global.cnki.net/

or please contact the author Dr. Maoan Han through email: mahan@sjtu.edu.cn

[8] J. Hofbauer And. J. W.-H. So, Multiple limit cycles for predator-prey models, Math. Biosci. 99 (1990), 71-75.

[9] Y. KuANG, Nonuniqueness of limit cycles of Gause-type predator-prey systems, Appl. Anal. 29 (1988), 269-287.

[10] Y. A. Kuznetsov, Elements of Applied Bifurcation Theory, Appl. Math. Sci. 112, Springer-Verlag, New York, 1995.

[11] R. M. MAY, Limit cycles in predator-prey communities, Science 177 (1972), 900-902.

[12] K. Mischaikow and G. S. K. Wolkowicz, A predator-prey system involving group defense: a connection matrix approach, Nonlinear Anal. 14 (1990), 955-969.

[13] N. Kasarinoff and P. VAn Der Driessche, A model of predator-prey system with functional response, Math. Biosci., 39 (1978), pp. 124-134.

[14] L. Perko, Differential Equations and Dynamical Systems, Springer-Verlag, New York, 1996.

[15] S. Pilyugin and P. Waltman, Divergence criterion for generic planar systems. SIAM J. Appl. Math. 64 (2003), no. 1, 81-93 (electronic).

[16] S. Pilyugin and P. Waltman, Multiple limit cycles in the chemostat with variable yield. Math. Biosci. 182 (2003), no. 2, 151-166.

[17] M. L. Rosenzweig, Paradox of enrichment: destabilization of exploitation ecosystems in ecological time, Science 171 (1971), 385-387.

[18] F. Rothe and D. S. Shafer, Multiple bifurcation in a predator-prey system with nonmonotonic predator response. Proc. Roy. Soc. Edinburgh Sect. A 120 (1992), no. 3-4, 313-347.

[19] S. RUAN AND D. XIAO, Global analysis in a predator-prey system with nonmonotonic functional response, SIAM J. Appl. Math. 61 (2001), 1445-1472.

[20] Waterloo Maple Software, MAPlE VII, University of Waterloo, Waterloo, Canada.

[21] G. S. K. Wolkowicz, Bifurcation analysis of a predator-prey system involving group defence, SIAM J. Appl. Math. 48 (1988), 592-606.

[22] D. M. Wrzosek, Limit cycles in predator-prey models, Math. Biosci. 98 (1990), $1-12$. 
[23] D. Xiao And Z. Zhang, On the uniqueness and nonexistence of limit cycles for predator-prey systems, Nonlinearity 16 (2003), 1185-1201.

[24] Z. ZhANG, On the existence of exactly two limit cycles for the Liénard equation, Acta Math. Sinica 24 (1981), 710-716 (in Chinese).

[25] Y. R. ZHOU, Existence and uniqueness of a limit cycle for the system of equations $\dot{x}=\varphi(y)-F(x), \dot{y}=-g(x)$, and the existence of exactly two limit cycles, Chinese Ann. Math. 3 (1982), 89-102 (in Chinese).

[26] H. Zhu, S.A. CAmpbell and G. Wolkowicz, Bifurcation analysis of a predatorprey system with nonmonotonic function response, SIAM. J. Appl. Math. 63 (2002), 636-682. 